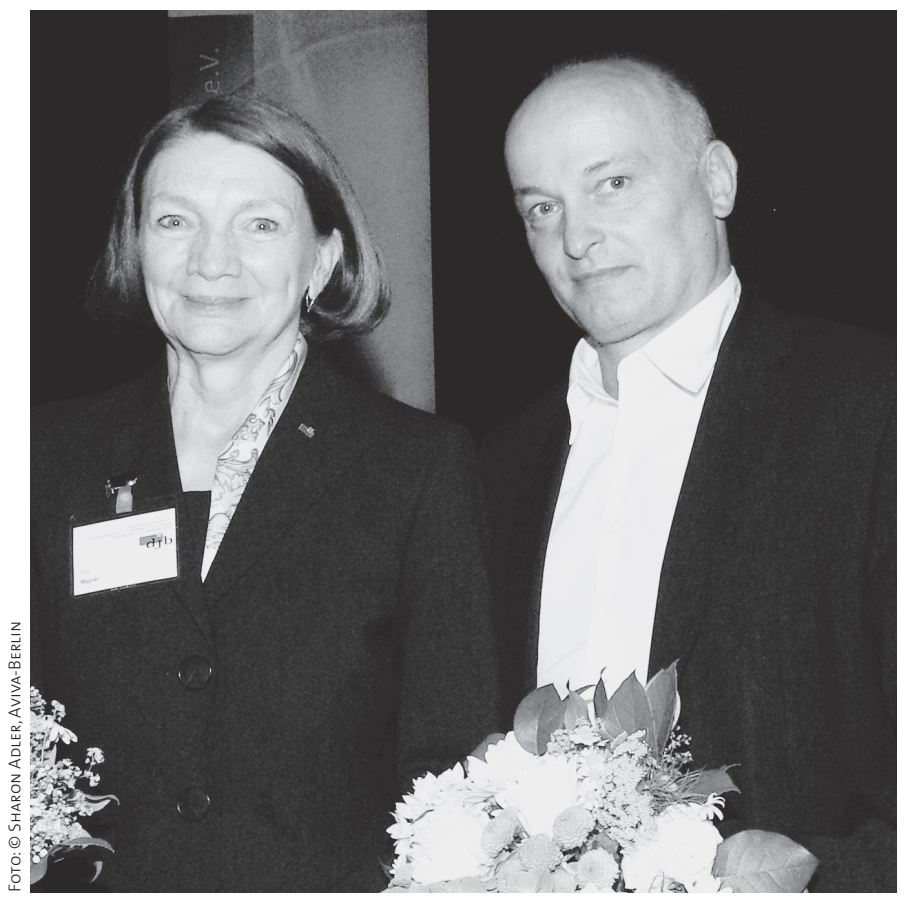

A Jutta Wagner überreicht Dr. Volkmar Schöneburg (Justizminister des Landes Brandenburg) als Dank für ein Grußwort an die Teilnehmerinnen und Teilnehmer des 39. Bundeskongresses einen Blumenstrauß.

nehmungen diskutiert. Es gab immer die zwei konträren Positionen: die einen, die sagten, man müsse die Unternehmen dazu zwingen, eine Quote durchzusetzen. Das ist auch meine Position. Außerdem hört man die mehr von freien Demokraten geäußerte Auffassung, dass man die Frauen in Führungs- positionen einem gewissen Selbstlauf überlassen müsse. Nein, ich denke, man muss dazu strukturelle Entscheidungen treffen, diese entsprechend fixieren und dann durchsetzen. Es ist schwer genug, wie die streitbaren Juristinnen gezeigt haben, die entsprechende Quote dann auch tatsächlich zu verwirklichen.

Eine zweite Anmerkung sei mir noch kurz gestattet. Ich freue mich sehr, dass es dem djb gelungen ist, die Ausstellung zu den Juristinnen aus der DDR auf die Beine zu stellen, die morgen Abend im Landgericht Potsdam eröffnet wird. Ich finde, dass ein differenzierter Blick auf Juristinnen in der DDR in zweierlei Hinsicht eine wichtige Sache ist: Man kann anhand der Ausstellung nachvollziehen, dass die Justiz in der DDR aus den unterschiedlichsten bekannten Gründen nicht so eine Rolle gespielt hat wie im Westen Deutschlands, dass sie eine Unterdrückungsfunktion hatte und die Strafjustiz hart war, dass zum Teil auch Unrecht in Rechtsform produziert wurde. Dafür steht das Porträt von Hilde Benjamin, wobei man auch sie nicht allein darauf reduzieren kann. Andererseits gelang der DDR eine bedeutend höhere Frauenquote unter den Juristen als parallel dazu der Bundesrepublik. Ich habe gestern Abend noch Uwe Wesels „Recht, Unrecht und Gerechtigkeit. Von der Weimarer Republik bis heute“ gelesen, der sagte, das habe auch mit dazu geführt, dass die Justiz im Westen etwas männlicher und härter und im Osten etwas weiblicher und außerhalb der politischen Konflikte auf mehr Harmonie ausgerichtet gewesen sei. Letzteres habe der Justiz gut zu Gesicht gestanden. Vielleicht kann man aus der Ausstellung auch dazu etwas lernen. Ich wünsche Ihnen eine wunderbare Tagung! Danke.

\title{
Gleichberechtigung ist ein Menschenrecht
}

\section{Dr. Max Stadler, MdB}

Parlamentarischer Staatssekretär bei der Bundesministerin der Justiz, Berlin

\section{Meine Damen und Herren,}

der Weg von Berlin aus dem Reichstag hierher war kurz, der Weg von dem sitzenden Publikum auf die Bühne ist relativ lang. Gleichwohl habe ich auch diesen Weg noch pünktlich geschafft und ich bedanke mich sehr herzlich für die Einladung, sehr geehrte Frau Präsidentin Wagner, sehr geehrter Herr Minister, Herr Oberbürgermeister, meine sehr geehrten Damen und Herren.

Eva Spagna, die Sängerin, die einen so schönen kulturellen Rahmen für die Veranstaltung bietet, meinte vorhin, beim Papst seien wahrscheinlich viele Männer gewesen. Das ist natürlich richtig, aber nur die halbe Wahrheit. Es waren auch viele Frauen dort. Manche haben sogar begeistert applaudiert. So zum Beispiel Claudia Roth und Renate Künast, weil der Papst zur allgemeinen Überraschung die ökologische Bewegung sehr gelobt hat. Ebenfalls war überraschend, dass er sich in seiner Rede vorhin im Reichstag mit rechtsphilosophischen Fragen befasste. Er hat sich auseinandergesetzt mit Hans Kelsen, der bekanntlich der Begründer der reinen Rechtslehre ist, der Schöpfer der österreichischen Verfassungsgerichtsbarkeit und ein bedeutender Rechtspositivist. Während der Papst sich als Verfechter des Naturrechts dargestellt hat. Ich glaube, egal welchen Begründungsstrang man verfolgt, eines steht fest: Gleichberechtigung ist ein unverbrüchliches Menschenrecht. Das werden Rechtspositivisten und Vertreter des Naturrechts gleichermaßen so sehen. Ich will mich nun in diese Fragen nicht weiter einmischen, denn wir hören jetzt gleich einen Vortrag von Frau Prof. Dr. Baer, die womöglich auch auf diese Themen eingeht, so dass ich es in meinem Grußwort dabei belassen will, einige wenige Fakten zu nennen, die zu Ihrem Thema passen. Deswegen, Frau Präsidentin, antworte ich jetzt auch nicht auf die rechtspolitischen Probleme, die Sie angerissen haben. Dafür ist ein Grußwort wohl nicht der richtige Ort.

Aber Ihr Kongress hat mir Anlass gegeben, im eigenen Haus und im eigenen Geschäftsbereich nachzuforschen, ob wir uns denn einigermaßen als Vorreiter für Gleichberechti- 
gung fühlen können. Dabei bietet die Hausleitung des Bundesministeriums der Justiz schon ein deutliches Signal, der Kollege Diwell weiß es aus seiner Zeit als Staatssekretär. Die Hausleitung besteht aus drei Personen. Es sind immerhin eine Ministerin und eine beamtete Staatssekretärin. Das ist schon eine gute Quote, die allerdings durch meine Anwesenheit als Parlamentarischer Staatssekretär dort im Hause ein wenig geschmälert wird. Wichtiger ist aber vielleicht die Auswertung unseres Personalverwaltungssystems, die wir gerade aktuell vorgenommen haben. Diese hat ergeben, dass der Frauenanteil in unserem Ministerium insgesamt stolze 58 Prozent beträgt. Im höheren Dienst sind es immerhin 44 Prozent. Und jetzt habe ich überlegt, ob ich den Rest meines Manuskriptes lieber streichen sollte, weil viele Präsidentinnen und Präsidenten der Gerichte anwesend sind, aber ich erwähne es doch: $\mathrm{Zu}$ unserem Geschäftsbereich gehören auch rund 240 Richterinnen und Richter an drei obersten Bundesgerichten, nämlich dem Bundesgerichtshof, dem Bundesverwaltungsgericht und dem Bundesfinanzhof. Hier fällt die Statistik folgendermaßen aus: Am Bundesgerichtshof sind von den 129 Richterstellen 28, also knapp 22 Prozent, mit Frauen besetzt. Bei den Vorsitzenden ist der Frauenanteil nicht einmal halb so hoch. Nur zwei von fünfzehn dieser Stellen sind mit Frauen besetzt. Beim Bundesverwaltungsgericht ist der Frauenanteil mit rund 26 Prozent zwar insgesamt höher, der Anteil bei den Vorsitzenden liegt derzeit allerdings bei null Prozent. Aber wir haben ja die große Freude, dass das Bundesverwaltungsgericht von einer hier heute anwesenden - Präsidentin geleitet wird, worüber ich mich sehr freue. Der Bundesfinanzhof schließlich hat mit 20 Prozent zwar den geringsten Frauenanteil insgesamt, aber ein Viertel der Vorsitzendenstellen ist mit Frauen besetzt. Das ist doch schon einigermaßen beachtlich.

Diese kurze Bilanz fällt also durchwachsen aus. Sie ist nicht ganz schlecht, aber sie ist auch noch nicht gut genug. Daher, Frau Präsidentin Wagner, will ich das Grußwort dazu nutzen, Ihnen zu versichern, Ihre Ankündigung von vorhin, dass Sie mit Ihrem Kongress, uns, dem öffentlichen Dienst als Arbeitgeber, schon die richtigen Instrumente empfehlen werden, nehme ich sehr ernst. Wir kommen gerne darauf zurück. Das gilt für die gesamte Hausleitung, für meine Person, der ich hier Gast sein darf, für die beamtete Staatssekretärin Frau Dr. Grundmann und für die Ministerin Frau LeutheusserSchnarrenberger, von der ich Sie sehr herzlich grüßen darf. Dankeschön.

\section{Es gibt viel zu tun - auch bei den Frauen in Leitungs- positionen des öffentlichen Dienstes}

\section{Jann Jakobs \\ Oberbürgermeister, Potsdam}

Sehr geehrte Frau Präsidentin Wagner, Herr Minister Dr. Schöneburg, Herr Staatssekretär Dr. Stadler, meine sehr verehrten Damen und Herren,

als Oberbürgermeister heiße ich Sie ganz herzlich willkommen zu Ihrem 39. Bundeskongress in der Landeshauptstadt Potsdam. Ich finde es richtig klasse, dass der Brandenburger Landesverband des djb diese Einladung ausgesprochen hat. Ich finde es auch toll, dass die Veranstaltung nicht etwa im Neuen Palais, im Cecilienhof oder womöglich in Sanssouci stattfindet, sondern hier in der Schiffbauergasse. Denn dieses Gelände dürfte weniger bekannt sein und ich finde es sehr schön, dass Sie sich hier heute in der Schinkelhalle versammeln. Ich finde es auch deshalb sehr erfreulich, weil Potsdam in der Regel mit wunderbaren Schlössern in Verbindung gebracht wird. Das ist auch gut so und eine gute Werbemarke. Aber es wäre wesentlich zu kurz gegriffen, wenn man Potsdam auf diese drei Bauwerke reduzieren würde. Insoweit haben wir ja auch dafür Sorge getragen, dass hier in der Schiffbauergasse ein Gegenakzent gesetzt wird - architektonisch, aber auch kulturell.

Hier versammelt sich auf der einen Seite die Kreativwirtschaft im VW Design Center. In Steinwurf-Nähe sagt man bes- ser nicht, weil das Center ein Glashaus in unmittelbarer Nähe ist. Auf der anderen Seite befindet sich die Deutschlandzentrale von Oracle. Diese Gewerbebetriebe in Verbindung mit Kultur ist etwas ganz Herausragendes. Wir haben hier unser Theater neu eröffnen können vor ein paar Jahren. Das hat wirklich Strahlkraft und wenn ich das sage, meine Damen und Herren, dann bedeutet das schon etwas in der unmittelbaren Umgebung Berlins. Und die Strahlkraft wirkt auch in Richtung Berlin. Ich bin immer sehr stolz darauf, wenn mir die Zahlen vorgelegt werden und ich sagen kann „Siehste, ein Drittel kommt aus Berlin“. Wir sagen hier, Berlin ist bedeutend, deshalb ist der Papst auch dort, aber Potsdam ist schön, deshalb sind Sie hier, meine Damen.

Potsdam zeichnet sich dadurch aus, dass es eine wachsende Stadt ist, eine der wenigen Städte im Osten Deutschlands, in Deutschland überhaupt, die wächst. Wir haben mittlerweile rund 157.000 Einwohner. Wenn man den Statistikern glauben darf, dann wird dieses Wachstum auch in den nächsten Jahren zu verzeichnen sein. Wir sollen in den nächsten zehn Jahren rund 170.000 Einwohner haben. Deshalb sind wir auch kräftig dabei, einen entsprechenden Wohnungsbau zu realisieren, damit die Einwohnerinnen und Einwohner dann auch alle hier wohnen können.

Der Tourismus ist eine ganz wichtige Einnahmequelle in dieser Stadt. Wir haben rund 900.000 Übernachtungen pro 